\title{
High frequency ventilation
}

High frequency ventilation (HFV) is a modality that exploded into the field of respiratory care over ten years ago, raising high expectations and then going on to leave many clinicians questioning whether anything of substance had really changed. A number of reviews are available, ${ }^{1-4}$

Unquestionably the ficld is confusing, largely because there is so little standardization of either equipment or usage. Rarely are two machines exactly the same, even in the hands of the same investigator, since "improvements" are always being incorporated. Frequently, ventilator design and function have been poorly characterized. Nevertheless certain patterns of observation now recur with enough consistency for us to distil a useful analysis We will focus on two questions:

1 Are HFV devices substantially different from conventional mechanical ventilators (CMV)?

2 Do the different types of high frequency ventilators differ significantly from each other?

\section{Classification of HFV}

\section{Rate}

One early definition of HFV was any form of ventilatory support operating at more than four times the normal resting breathing frequency of that species. This definition spanned the wide range from the high frequency positive pressure ventilation (HFPPV) of Sjöstrand and colleagues at $60 \mathrm{bpm},{ }^{5}$ to the high frequency oscillatory (HFO) modes operating at 300-3000 breaths per minute (bpm). ${ }^{3}$

More recently, distinctions based on gas transport mechanisms have been proposed. ${ }^{6}$ Analyses of gas transport in a variety of species identify a transition frequency below which gas transport can be accounted for by our classic equations where $V_{A}=\left(V_{T}-V_{D}\right) f$, and above which transport must be related to the complex mixing processes that have been discussed for $\mathrm{HFV}^{7}{ }^{7}$ This tratsition frequency varies with species, being $-170 \mathrm{bpm}$ in the normal human lung. ${ }^{6}$ In light of this analysis, we may need to rethink our use of the term HFV, recognizing that some techniques may be better thought of simply as "rapid CMV," with the term HFV being reserved for those modalities that operate in the domain of HFV transport mechanisms.
High or low driving pressure

Another potential classifying feature could be the driving pressure of the various HFV devices. High frequency jet ventilators (HFJV) and high frequency flow interrupters (HIFI) use high pressure ( $\sim 50 \mathrm{psi})$ gas sources whose output is chopped into brief flow pulses by some type of valving mechanism. Such devices have sufficient power to drive the desired stroke volume through either a regular endotracheal tube or a long narrow cannula. ${ }^{4}$ High frequency oscillators (HFO) are generally reciprocating pumps of a bellows, diaphragm, or piston type that generate an approximately sinusoidal flow of gas in and out of the lungs with much lower driving pressures. ${ }^{3}$ Therefore, existing HFO devices generally are attached directly to the endotracheal tube and may not work well through a narrow cannula. This feature needs to be considered for certain surgical/diagnostic applications such as bronchoscopy.

\section{Active or passive expiration}

Experience has shown that the nature of expiratory flow is a significant functional property of HFV devices. Devices that depend on passive recoil of the lung and chest wall to drive gas out of the lungs (e.g., HFJV or HIFI) are prone to develop pulmonary hyperinflation with increasing frequency. This phenomenon has been termed gas trapping, auto-PEEP, or inadvertent PEEP. It is often accompanied by inadequate $\mathrm{CO}_{2}$ elimination \pm cardiac output depression. The extent of hyperinflation depends on the duration of expiration plus the mechanical properties of the lungs. ${ }^{B}$

Devices with active expiration (i.e., the oscillators) are much less prone to hyperinflation because the ventilator actively pulls gas out of the system on each expiration. Although some hyperinflation can occur with certain types of HFO circuits, particularly at low mean airway pressures, it is much less common than with high frequency devices with passive expiration (i.e., HFV-P). ${ }^{9}$ Therefore high frequency ventilator a with active expiration (HFV-A) can be used at rates of $15-30 \mathrm{~Hz}(900-3000 \mathrm{bpm})$, unlike HFV-P devices

Departments of Anaesthesia, Paediatrics and Physiology, Quccn's University, Kingston General Hospital, Kingston, Ontario, K7L 3N6. 
Recently, hybrids and subtle variants have appeared Some users have tried to add active expiration to classic HFJV. In one report, valving in a circuit produced an oscillator with passive expiratory flow. Since the nature of expiratory fow appears to influence HFV performance strongly, it should be clearly specified.

\section{Is HFV better than CMV?}

Are high frequency ventilators merely novel, or can they truly handle some problems better than our existing conventional ventilators? This question can be asked with reference to a variety of specific areas in which improved ventilator performance may be sought.

\section{Surgical procedures}

Many things $c a n$ be done using HFV. Whether they need to be done that way is often questionable. For example, laryngoscopy, bronchoscopy, tracheal reconstruction over a T-tube, or sleeve pneumonectomies can be managed well using HFJV, but they can also be handled well with normal frequency jet ventilation. The key requirement is the ability to ventilate through a small cannula under conditions in which a tight seal between endotracheal tube and airway wall is impossible.

More recently HFJV has been used successfully during extracorporeal shock wave lithotripsy (ECSWL) to maintain gas exchange with minimal respiration-related stone motion. Again one must remember that ECSWL can also be managed using appropriately coordinated $\mathrm{CMV}$, or even regional anaesthesia. The assessment of relative risks and benefits of any given approach must include awareness of the added safety precautions needed for HFV

Laser resection of bronchial tumours is one situation in which having a near-motionless operative field may be of significant advantage to the endoscopist, but again HFV is not essential. A number of clinical trials of HFV during thoracotomy and other surgical procedures have established that it is as effective as CMV in maintaining gas exchange. However, persistent inflation of the nondependent lung during HFV may impede surgical access.

\section{$\mathrm{CO}_{2}$ elimination}

The fact that gas transport can be adequate even with a stroke volume that is only a fraction of anatomical deadspace has forced us to rethink our traditional conecpts of gas exchange. The transport mechanisms of HFV have been well described by Chang. ${ }^{7}$ Transport analyses have all been performed using the sinusoidal waveforms of HFO (i.e., HFV.A device). A variety of mixing mechanisms contribute to transport, most of which depend on high gas velocities, and therefore the high rates of HFV.
The transition to HFV transport mechanisms occurs approximately at $\mathrm{VT}<1.2 \mathrm{VD}$ (where $\mathrm{VD}=$ anatomical + equipment deadspace). ${ }^{6}$ With HFV-A devices, no clear optimum frequency for $\mathrm{CO}_{2}$ transport exists. HFV-P devices, however, generally become ineffective at frequencies $>200-300 \mathrm{bpm}$.

If $\mathrm{CO}_{2}$ retention is the primary limiting problem, then HFV will often succeed where CMV is failing. Both HFV-A ${ }^{10}$ and HFV-P ${ }^{11}$ devices have proved successful under such circumstances. HFV may offer significant advantage if one needs to move $\mathrm{CO}_{2}$ at lower peak and mean airway pressures than are required for CMV. (Note that although HFV is pressure-efficient it is not minutevolume efficient. Minute flows during HFV are often many times greater than CMV.) HFV may be useful to manage pulmonary interstitial emphysema in infants, massive airleak from bronchopleural-cutaneous fistulae, or persistent pulmonary hypertension of the newborn. However, HFV may not be successful in these applica. tions if coexistent parenchymal disease (such as diffuse atelectasis) necessitates use of higher mean airway pressures to support oxygenation.

\section{Oxygenation}

Since HFV transports $\mathrm{CO}_{2}$ so effectively, hypoxia that is secondary to $\mathrm{CO}_{2}$ retention on CMV often will improve on HFV. If hypoxia arises from extensive low, but nonzero $\dot{V}_{A} / Q$ regions, the answer remains unclear. Although the unusual mixing mechanisms of HFV transport would suggest that intrapulmonary gas distribution might be more even on HFV than CMV, there is no rigorous proof that such an effect occurs. However, since hypoxia secondary to low $\dot{V}_{A} / Q$ regions responds readily to increases in $\mathrm{FIO}_{2}$, this is not a major area of concern.

In the presence of intrapulmonary shunt (as with the diffuse atelectasis of adult respiratory distress syndrome, or ARDS) oxygenation on HFV exhibits the same dominant dependence on mean transrespiratory distending pressure as does CMV. Many early reports of "improved oxygenation" on HFV merely reflect the systematic use of higher mean airway pressures during HFV.

In order for HFV to offer any substantial benefits over CMV with respect to oxygenation, one would need to find a method to obtain a better $\mathrm{PaO}_{2}$ for any given mean airway pressure, by somehow achieving a larger lung volume at that applied pressure. Such an idea is not novel. It has been tried before with CMV with various "sigh" modes, and subsequently abandoned. The concept has been based on knowledge that the pressure volume curve of even a sick lung generally exhibits some hysteresis, such that lung volume is greater for a given applied pressure on the "deflation limb" of the pressurevolume (P/V) curve, after the lung has been taken up 
near total lung capacity for a brief period. With CMV the "sigh" proved useless. Any volume gained was lost at the end of the next expiration. It proved better to use PEEP plus larger tidal volumes and slower rates.

It is in this area that high frequency ventilators may prove to have some tactical advantage over CMV, simply because the pressure/time profile is distinctly different with HFV. High rates and small pressure/volume cycles improve one's chances of holding the atelectasis-prone lung above the point on the P/V curve at which alveolar airway closure occurs, since during HFV at high rates $\mathrm{CO}_{2}$ transport can be sustained with cyclic volume changes of only $1-2 \mathrm{ml} \cdot \mathrm{kg}^{-1}$ around the mean value.

These concepts have been explored extensively for HFO. Brief $(\sim 15 \mathrm{sec})$ sustained inflations to a pressure $10-15 \mathrm{~cm} \mathrm{H}_{2} \mathrm{O}$ above the maintenance mean airway pressure often yield substantial improvements in mean lung volume and oxygenation, both in animul models of lung disease and in humans. ${ }^{12,13}$ This approach has not been systematically investigated for other HFV devices. Empirically, a number of combined forms of HFV have evolved that incorporate a periodic large breath of some kind, of variable duration and frequency. These large breaths appear to engender some type of volume recruitment and improve oxygenation. More study is needed in this area to assess the relative efficacy, appropriate operating frequency, and optimal protocol for volume recruitment with the multiple HFV devices currently available. Timing of intervention may prove to be an important limitation to the use of HFV volume recruitment techniques. Responsiveness is greatest early in the disease course. Once structural injury is advanced and hysteresis is minimal, volume recruitment manoeuvres are ineffective.

\section{Lung injury}

A major stimulus for the development of $\mathrm{HFV}$ has been the desire to decrease ventilator-related lung injury, particularly in treatment of premature infants with RDS. Progress in this area has been hampered by a basic lack of understanding of mechanisms of lung injury. Is it high oxygen, high pressure, overdistension, or tissue fragility that exaccrbates the process?

This ignorance of fundamental mechanisms has generated ventilator protocols that pursue contradictory goals. Some studies give top priority to the use of low ventilator pressures; others give priority to achieving maximal alveolar reexpansion. In general randomized studies showing a reduction of lung injury on HFV ys CMV have utilized protocols that achieved higher lung volumes on HFV than in the CMV-treated groups. A recent study by McCulloch et al. is important in this regard. ${ }^{14}$ In this study of atelectasis-prone rabbits, lung injury was only prevented by the combination of HFO and high lung volume. HFO with ongoing atelectasis (i.e., lower pressures) produced an injury pattern approaching that of CMV. Again timing of intervention appears to be a key factor. Many of the benefits reported with HFV only occur when intervention occurs before significant structural damage has developed.

\section{Problems engendered by HFV}

\section{Monitoring}

Many of our usual monitoring approaches (such as expired tidal volume, end tidal $\mathrm{CO}_{2}$ monitors, or circuit pressures) do not work well at high ventilator rates. New approaches must be devised and tested for each new system. Accurate pressure monitoring is essential, but not easy. During many early HFJV trials, operators telied on pressure measurements in the circuit or at the top of the endotracheal tube. Such sites yield gross underestimates of intrapulmonary pressure. Pressure must be sampled at the tip, or preferably beyond the tip of the endetracheal tube during HFV-P, or else a lethal buildup of intrapulmonary pressure can go unrecognized and uncorrected. Pressure gradients between proximal and distal sites tend to be smaller during HFV-A. However, any pressure monitoring system must be thoroughly tested and understood before being used clinically.

Online monitors of oxygenation have proven extremely valuable during $H F V$, especially when using the very high rates of HFO. With high rates and small volumes, visual estimation of the adequacy of stroke volume becomes more difficult. The assessment of vital signs is also more difficult during HFV. Auscultation of heart rate or heart sounds is often impossible, increasing dependence on electronic monitoring devices.

\section{Safety}

Automatic recognition and cortection of malfunctions is essential when using the high ventilator rates of HFV. An alarm mode alone is not enough. For example, the pressure monitoring system during HFJV must automatically shut off driving pressure whenever the high pressure limit is exceeded. System modifications must be made very cautiously and tested over the entire range of conditions in which the system may be used. For example, suctioning during HFJV may be safe and advantageous under certain conditions, but it has also been associated with lethal barotrauma. ${ }^{15}$

\section{Humidification}

Humidification has proven difficult during HFJV, and must be approached with care. Serious tracheal mucosal injury has been reported, ${ }^{16}$ some of which appears related to inadequate humidification. Oscillator-type devices 
pose less problems since the minute flows of gas that need to be humidified are lower than with HFJV, and pass through the humidifier at a constant flow rather than in brief pulses.

\section{Bronchospasm}

Every large series has one to two patients in whom prohibitive degrees of bronchospasm developed when they were placed on HFV, as opposed to CMV. This has occurred with both HFV-P and HFV-A devices.

\section{Summary}

The potential role of HFV has yet to be fully defined. Its use should be approached with caution, ensuring that the possible benefits outweigh new hazards. Only further rigorously controlled trials, designed to make optimum use of the special features of HFV, will fumish the information we need.

\section{References}

1 Froese $A B, B r y a n A C$. High frequency ventilation. Am Rev Respir Dis (April 1987).

2 Drazen $J M, K a m m R D$. Slutsky $A S$. High-frequency ventilation. Physiol Rev 1984; 64: 505-43.

3 Kolton $M$. A review of high frequency oscillation. Can Anaesth Soc J 1984; $31: 416-29$.

4 Carlon GC, Miodownik S, Ray C Jr. High-frequency jet ventilation: technical evaluation and device characterization. In: Carlon GC, Howland WS (Eds). High-frequency ventilation in intensive care and during surgery. New York: Marcel Dekker, $1985 ; 77-110$.

5 Sjöstrand $U$. High-frequency positive-pressure ventilation (HFPPV): a review, Crit Care Med 1980; 8: 345-64.

6 Venegas JG, Hales CA, Strieder DJ. A general dimensionless equation of gas transport by high-frequency ventilation. J Appl Physiol 1986; 60: 1025-30.

7 Chang $H K$. Mechanisms of gas transport during ventilation by high-frequency oscillation. J Appl Physiol 1984; 56: 553-63.

8 Rouby $J$, Simonneau $G$, Benhamou $D$ et al. Factors influencing pulmonary volumes and $\mathrm{CO}_{2}$ climination during high-frequency jet ventilation. Anesthesiology 1985; 63: 473-82.

9 Bryan AC, Slutsky AS. Lung volume during high frequency oscillation. Am Rev Respir Dis 1986; 133: 928-30.

10 Frantz ID III, Wershammer J, Stark AR. High-frequency ventilation in premature infants with lung disease: adequate gas exchange at low tracheal pressure. Pediatrics 1983; 71: 483-8.

11 Turnbull AD, Carlon G, Howland WS, Beatie EJ. Highfrequency jet ventilation in major airway or pulmonary disruption. Ann Thorac Surg 1981; 32: 468-74.
12 Kolton $M$, Cattran $C B$, Kent $G$, Volgyesi $G$, Froese $A B$, $B$ ryan $A C$. Oxygenation during high-frequency ventilation compared with conventional mechanical ventilation in two models of lung injury. Anesth Analg 1982; 61: 323-32.

13 Froese $A B$, Butler $P O$, Fletcher WA, Byford LJ. High frequency oscilatory ventilation in premature infants with respiratory failure: a preliminary report. Anesth Analg (In Press).

14 McCulloch PR, Forkert PG, Froese AB. The tole of lung volume maintenance during high frequency oscillatory ventilation (HFO-A) in surfactant deficient rabbits. Am Rev Respir Dis 1986; 133: A154.

15 Egol A, Culpepper JA, Snyder JV. Barotrauma and hypotension resulting from jet ventilation in critically ill patients. Chest 1985; 88: 98-102.

16 Ophoven JP, Mammel MC, Gordon MJ, Boros SJ. Tracheobronchial histopathology associated with high-frequency jet ventilation. Crit Care Med 1984; 12: 829-32. 